\title{
Luminosity determination for the proton-deutron reactions using quasi-free reactions with WASA-at-COSY facility
}

\author{
Aleksander Khreptak ${ }^{1, *}$, Oleksandr Rundel ${ }^{1}$, and Magdalena Skurzok ${ }^{1}$ \\ for the WASA-at-COSY Collaboration \\ ${ }^{1}$ Marian Smoluchowski Institute of Physics, Jagiellonian University, Kraków, Poland
}

\begin{abstract}
The scientific aim of our research is experimental confirmation of the existence of mesic-nucleus: a new exotic kind of nuclear matter consisting of nucleons and mesons. In this report we present the method of luminosity determination for experiment performed at WASA-at-COSY detector dedicated to search for ${ }^{3} \mathrm{He}-\eta$ bound state. This method is based on analysis of quasi-free $p d \rightarrow p p n_{\text {spec }}$ reaction.
\end{abstract}

\section{Introduction}

In 1986, Haider and Liu [1] postulated the hypothesis that the neutral mesons such as $\eta$ or $\eta^{\prime}$ can form bound states with atomic nuclei due to the strong interaction. Since then, experimental searches have been performed by several experiments [2-13], however, so far there is no direct experimental confirmation of the existence of mesic nuclei.

In 2014, we performed a search for the $\eta$-mesic ${ }^{3} \mathrm{He}$ with the WASA detector installed at the Cooler Synchrotron COSY at the Jülich Research Center (Germany) in proton-deuteron collisions at energies around the $\eta$ production threshold [14]. In the experiment, the proton beam momentum was varied continuously from $1.426 \mathrm{GeV} / \mathrm{c}$ to $1.635 \mathrm{GeV} / \mathrm{c}$, what corresponds to the excess energy $Q \in(-70,30) \mathrm{MeV}$. During a ramping process the luminosity could vary due to displacement of the beam at the target position correlated with the variation of the momentum and adiabatic shrinking of the beamsize [15]. For this reason it is crucial to determine luminosity dependence on the excess energy for the normalization of the obtained excitation function for studied reactions and for interpretation of the results in view of the hypothesis of the ${ }^{3} \mathrm{He}-\eta$ bound state production.

\section{Quasi-free $p p \rightarrow p p$ reaction studies}

In order to determine the luminosity dependence on the excess energy $Q$, we used the quasielastic proton-proton scattering process and the method discribed in Ref. [16, 17]. In the proton-deuteron collisions, the protons from the beam scatter on the protons in the deuteron target and the neutrons from the deuteron play a role of spectators: $p d \rightarrow p p n_{\text {spec }}$.

The Monte Carlo simulations of this reaction were carried out. The proton beam momentum was generated with uniform probability density distribution in the range corresponding to the experimental ramping. The target neutron $n_{t}$ momentum vectors are distributed

\footnotetext{
*e-mail: aleksander.khreptak@doctoral.uj.edu.pl
} 
isotropically in the spherical coordinates inside deuteron $\left(p_{F_{t}}, \theta_{F_{t}}, \phi_{F_{t}}\right)$ with Fermi momentum distribution of nucleons inside deuteron $[18,19]$. The proton-proton invariant mass $\sqrt{s_{\mathrm{pp}}}$ was determined and the effective proton beam momentum $p_{\text {beam }}^{\mathrm{pp}}$ was calculated in the frame where proton $p_{t}$ inside deuteron is at rest. The scattering $\theta^{*}$ and the azimutal angle $\phi^{*}$ of the protons in the center-of-mass frame were generated isotropically. Four-momenta of outgoing particles were transformed to the laboratory frame and were used as an input in the simulation of the detection system response. Each of $N_{0}$ simulated event was weighted by the differential cross section for the quasi-free $p d \rightarrow p p n_{\text {spec }}$ reaction which is a function of the scattering angle $\theta^{*}$ and the effective proton beam momentum $p_{\text {beam }}^{\mathrm{pp}}$. We computed it based on the SAID partial-wave program [20]. Differential cross sections for proton-proton elastic scattering are presented in Fig. 1.

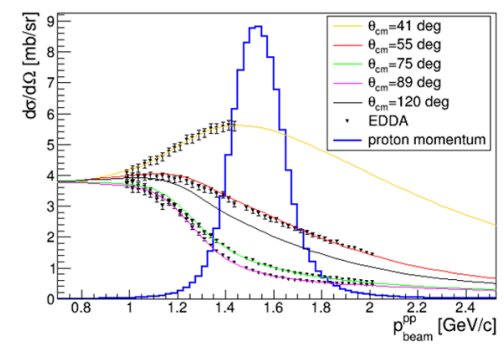

Figure 1. Differential cross sections for proton-proton elastic scattering as a function of the effective beam momentum for different values of the scattering angle in the CM frame. Triangles show EDDA collaboration data [21]. Lines denote SAID calculations [20]. Blue line presents distribution of the effective beam momentum obtained from simulations.

\section{Luminosity calculation}

In order to select quasi-free $p p \rightarrow p p$ events the condition of exactly one charged particle in the Forward Detector (FD) and one particle in the Central Detector (CD) was applied. Then, the dominating background processes $p d \rightarrow d \pi^{+} n_{\text {spec }}$ and $p d \rightarrow p d$ were subtracted. The events corresponding to the charged pions registered in the $\mathrm{CD}$ were subtracted based on energy deposited in the electromagnetic calorimeter and thin plastic scintillator (left panel of Fig. 2), while the background coming from elastic $p-d$ scattering was subtracted applying the cut in polar angle $\theta_{C D}$.

After all selection cuts and conditions the luminosity was calculated according to formula:

$$
L=\frac{N_{0_{i}}}{2 \pi} \frac{N_{\exp _{i}}}{N_{W M C_{i}}}
$$

where $N_{0_{i}}$ is the number of simulated quasi-free $p d \rightarrow p p n_{\text {spec }}$ events, $N_{\text {exp }_{i}}$ - the experimental number of scattered events after all applied cuts and $N_{W M C_{i}}$ - the number of events obtained from MC simulation after all applied cuts weighted with the values of the differential cross section for $p-p$ elastic scattering. Index $i$ means $i$ 'th of 40 intervals of excess energy $Q$.

The obtained total integrated luminosity is equal to $L=\left(2295 \pm 3_{\text {stat }} \pm 91_{\text {syst }}\right) n b^{-1}$. The luminosity was fitted by 4 degree polynomial $a Q^{4}+b Q^{3}+c Q^{2}+d Q+e$. The fitted function is shown in right panel of Fig. 2. 

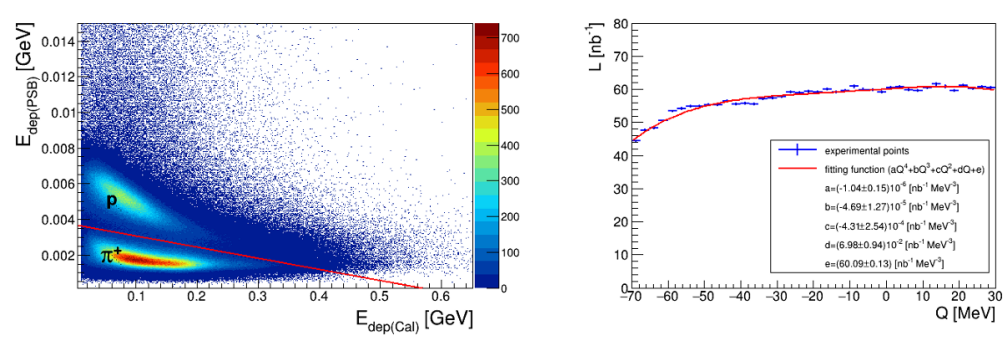

Figure 2. Left: experimental spectra of the energy loss in the Central Detector. The applied cut is shown as a red line. Right: Luminosity calculated for quasi-free $p d \rightarrow p p n_{\text {spec }}$ reaction (blue points) with fitted 4 degree polynomial function (red line)

\section{Summary}

We determined the luminosity and its dependence on the beam momentum for the experiment performed with WASA-at-COSY detector to search for the ${ }^{3} \mathrm{He}-\eta$ bound states in proton-deuteron fusion. The luminosity was calculated based on quasy-free $p d \rightarrow p p n_{\text {spec }}$ reaction and is equal to $L=\left(2295 \pm 3_{\text {stat }} \pm 91_{\text {syst }}\right) \mathrm{nb}^{-1}$.

We acknowledge the support from the Jagiellonian University through grant DSC 2016 for financing young scientist and PhD students No. 142/F/OR/2016, grant DSC 2017 No. K/DSC/004360, grant DSC 2018 No. K/DSC/004984 and from the Polish National Science Center through grants DEC2013/11/N/ST2/04152 and 2016/23/B/ST2/00784.

\section{References}

[1] Q. Haider, L. C. Liu, Phys. Lett. B 172, 257 (1986)

[2] T. Mersmann et al., Phys. Rev. Lett. 98, 242301 (2007)

[3] J. Smyrski et al., Phys. Lett. B 649, 258 (2007)

[4] A. Budzanowski et al., Phys. Rev. C 79, 012201 (2009)

[5] Y. K. Tanaka et al., Phys. Rev. Lett. 117, 202501 (2016)

[6] P. Moskal, J. Smyrski, Acta. Phys. Pol. B 41, 2281 (2010)

[7] S. V. Afanasiev et al., Phys. Part. Nucl. Lett. 8, 1073 (2011)

[8] H. Fujioka, Acta Phys. Pol. B 41, 2261 (2010)

[9] B. Krusche et al., J. Phys. Conf. Ser. 349, 012003 (2012)

[10] F. Pheron et al., Phys. Lett. B 709, 21 (2012)

[11] M. Skurzok, P. Moskal, W. Krzemień, Prog. Part. Nucl. Phys. 67, 445 (2012)

[12] P. Adlarson et al., Phys. Rev. C 87, 035204 (2013)

[13] P. Adlarson et al., Nucl. Phys. A 959, 102 (2017)

[14] O. Rundel, M. Skurzok, O. Khreptak, P. Moskal, Acta Phys. Pol. B 48, 1807 (2017)

[15] M. Skurzok, P. Moskal, W. Krzemień, Acta. Phys. Pol. B 46, 133 (2015)

[16] P. Moskal, R. Czyzykiewicz, AIP Conf. Proc. 950, 118 (2007)

[17] P. Moskal et al., Phys.Rev. C 79, 015208 (2009)

[18] M. Lacombe et al., Phys. Lett. B 101, 139 (1981)

[19] R. Machleidt et al., Phys. Rev. C 63, 024001 (2001)

[20] The CNS Data Analysis Center: http://gwdac.phys.gwu.edu/

[21] D. Albers et al., Phys. Rev. Lett 78, 1652 (1997) 